\title{
Using sewage for surveillance of antimicrobial resistance
}

\section{Aarestrup, Frank Møller; Woolhouse, Mark E.J.}

\section{Published in:}

Science

Link to article, DOI:

10.1126/science.aba3432

Publication date:

2020

Document Version

Early version, also known as pre-print

Link back to DTU Orbit

Citation (APA):

Aarestrup, F. M., \& Woolhouse, M. E. J. (2020). Using sewage for surveillance of antimicrobial resistance. Science, 367(6478), 630-632. https://doi.org/10.1126/science.aba3432

\section{General rights}

Copyright and moral rights for the publications made accessible in the public portal are retained by the authors and/or other copyright owners and it is a condition of accessing publications that users recognise and abide by the legal requirements associated with these rights.

- Users may download and print one copy of any publication from the public portal for the purpose of private study or research.

- You may not further distribute the material or use it for any profit-making activity or commercial gain

- You may freely distribute the URL identifying the publication in the public portal

If you believe that this document breaches copyright please contact us providing details, and we will remove access to the work immediately and investigate your claim. 


\title{
Using sewage for surveillance of antimicrobial resistance: A global system would exploit metagenomic sequencing.
}

\author{
Frank M. Aarestrup ${ }^{1}$ and Mark E. J. Woolhouse ${ }^{2}$
}

${ }^{1}$ Technical University of Denmark, DK-2800 kgs. Lyngby, Denmark. ${ }^{2}$ Usher Institute, University of Edinburgh, King's Buildings, Edinburgh, EH9 3FL, UK.

Email: Mark.Woolhouse@ed.ac.uk

Antimicrobial resistance (AMR), a cross-cutting and increasing threat to global health (1-3), is a complex problem with multiple and interconnected drivers. Reliable surveillance data that accurately describes and characterizes the global occurrence and distribution of AMR is essential for: tracking changes in resistance over time; national and global priority setting; assessing the impacts of interventions; identifying novel kinds of resistance; and supporting investigation of (international) outbreaks of resistant pathogens. AMR surveillance data can also inform development of treatment guidelines. Yet it has proven difficult to achieve these objectives on a global scale, and especially in lower and middle income countries (LMICs), in large part because current surveillance systems deliver data that is extremely variable in quality and quantity and highly heterogeneous in terms of which population is sampled (usually a category of hospital patients) and what drug-bug combinations are included (1). Here, we outline a plan for a global AMR surveillance system based on applying next generation sequencing (NGS) to human sewage that will be especially helpful for community AMR surveillance, which is difficult to achieve in other ways, and will provide an affordable surveillance option in resource-poor settings.

NGS is a powerful technology that has transformed the health data landscape. Among many other benefits, it has drastically improved our ability to determine the presence of AMR genes (bacterial genes known to confer resistance to an antimicrobial drug) in single isolates and to quantify them in complex microbiomes $(4,5)$. Millions of random DNA fragments sequenced using NGS can be mapped to reference sequence databases and the number of reads coming from any of several thousand known AMR genes can be counted to provide easily shared information on their occurrence and abundance.

Increasing numbers of people globally are connected to sewage treatment systems (6) and, as recently highlighted by the World Bank (3), metagenomics-based, near real-time quantification of AMR genes in sewage is a potentially useful surveillance tool even in remote locations without microbiology laboratories (7). Such an approach could quickly plug current gaps in the geographic, population, and agent coverage of AMR surveillance, especially by providing data on AMR outside hospitals ( $90 \%$ of antibiotic usage (in humans) occurs outside hospitals (Fig. 1)). It could also provide information on environmental transmission in populations exposed to raw sewage.

\section{Current global AMR surveillance}

The relevance of local and national surveillance of AMR to inform treatment guidelines and intervention strategies has been recognized for decades. The first international AMR-surveillance program was The European Antimicrobial Resistance Surveillance Network (EARS-Net), whose predecessor (EARS) was launched in 1998. EARS-Net is based on routine clinical antimicrobial susceptibility data from clinical laboratories reported to the European Centre for Disease Control and Prevention (ECDC). Only data from invasive isolates (blood and cerebrospinal fluid) and for seven bacterial pathogens are included. 
The Global Antimicrobial Resistance Surveillance System (GLASS) was launched in October 2015 by the World Health Organisation (WHO) to support their global action plan on AMR. A number of local WHO surveillance networks had already been established prior to GLASS and AMR data were also included in surveillance of single pathogens such as Mycobacterium tuberculosis and Neisseria gonorrhoea. As of January 2020 GLASS had enrolled 90 countries covering all regions (though not all have yet provided data), each reporting on up to eight different pathogens and up to 35 drug-bug combinations considered the most clinically important (though often only a small subset of these). In addition to these formal systems, a number of more informal AMR surveillance initiatives have been established, such as ResistanceOpen that provides online maps of the occurrence of 4 "super-bugs" worldwide, and ResistanceMap that maps resistance data for 12 bacterial pathogens from 46 countries.

A common feature of all these initiatives is that they focus on hospitalised patients and mainly last resort antimicrobial agents such as carbapenems (used after other agents have proven ineffective)(Fig. 1). This reflects the clinical perception that resistance to last resort antibiotics is most critical for patients, and the ease of access to clinical diagnostic facilities, put in place to improve patient outcomes and not, primarily, to facilitate AMR surveillance. This emphasis on clinical settings makes it difficult to determine the global spread of resistance to first-line drugs in the wider community, a large part of the global AMR burden (8). Indeed, it has recently been argued that interventions to support first-line drugs (e.g. tetracyclines) might have much greater public health impact than against last resort antimicrobial agents (8), the argument being that if the initial treatment works, the patients will never need a last resort antimicrobial treatment.

Because current isolate-based surveillance greatly relies on testing already being conducted for clinical purposes, it is often based on small sample sizes and can be biased. Nor is it easily implemented in resource-poor settings where there are no laboratories to perform bacterial isolation, identification and susceptibility testing and only a subset of the population may have access to clinical diagnostics. In addition, it has proven difficult to coordinate and harmonize both sampling and susceptibility testing results: different definitions for clinical cases may be used, methods for identification differ and different antimicrobial agents are tested.

\section{Benefits and limitations of sewage-based surveillance}

Examination of sewage inlets to treatment plants is already recommended for polio surveillance and, more recently, sewage has been successfully used for quantifying the occurrence and abundance of AMR genes in human populations (4,5,9-11). These studies mostly used metagenomic sequencing (which can detect all known resistance genes), though sometimes qPCR (which tar gets only selected genes). Even a single sample from one site can be representative of a large, urban population and a complete profile (occurrence and prevalence) of 1,000s of AMR-genes (the resistome) within that population can be obtained (4). In addition, some studies have suggested that resistance data from sewage can correlate well with data from clinical surveillance $(10,11)$.

However, global sewage-based surveillance using metagenomics differs from conventional measures of levels and burden of AMR in several key respects. It generates pooled data from a large, non-hospital population (whereas most surveillance data refer only to hospital patients). The data are also pooled across all bacteria taxa (i.e., do not refer to a subset, usually cultured bacterial pathogens). It measures AMR gene frequencies (not the prevalence of phenotypic resistance in a collection of isolates). Sewage therefore provides a different measure of AMR obtained using 
a different sampling frame and as such can augment current surveillance based on clinical isolates. Ideally, data from these two sources would be collected in parallel, allowing calibration and confirmation of geographic patterns and temporal trends.

We recognise certain limitations. Sewage-based AMR metagenomic surveillance, unlike isolatebased surveillance, does not link the AMR genes to specific bacterial species (though it can be argued that for surveillance purposes it is the genes that are of interest). Also, sensitivity is likely lower than isolate-based surveillance (though this may be compensated for by not being limited to a few bacterial species):

At the same time, however, sewage based metagenomic AMR surveillance has several important advantages. It characterises large communities that are not routinely assessed using conventional surveillance (though only those connected to the sewage system). It is straightforward to implement; at its most basic only requiring sample collection (using inexpensive equipment that is readily available) and shipment. Sequencing and bioinformatics methods are easily standardised, especially if done by a central facility. Ethical concerns have not been raised and there is no legal requirement for informed consent as data cannot be linked to any individual (4). It is not limited to an often very restricted subset of drug-bug combinations. It can provide a baseline for future trends and to monitor the effects of interventions in any location, irrespectively of whether the diagnostic capacity to isolate and identify bacterial pathogens exists. In the absence of good clinical surveillance it can provide a comparison of countries/regions, indicating where further actions are needed. When based on metagenomics it allows for retrospective analyses of data should novel genes subsequently be identified, providing a rapid assessment of global emergence. In addition, metagenomics provides information on all DNA and potentially RNA in the sample and can thus also be useful for surveillance of any living organisms, including enteric pathogens (12).

Sewage-based surveillance is also relatively cheap. For example, the World Bank has estimated the annual cost for clinical, isolate-based surveillance in one LMIC - Kenya - at approximately US\$2M (2). From our own experience (4), we estimate that the additional costs for collection, shipment, DNA purification, sequencing, and bioinformatics analysis of two sewage samples annually from two sites within the same country would be less than $0.1 \%$ of this sum. We therefore consider sewage-based surveillance to be a potentially valuable addition to current options for global AMR surveillance and monitoring. Though not a substitute for other surveillance methods it can provide data that is otherwise hard to obtain, and may sometimes be the easiest route to providing any information at all, especially in resource-poor settings.

\section{Necessary steps to make this happen}

Two important considerations are the DNA-purification methodology and the choice of bioinformatics analyses, both of which can influence the outcome. Protocols for sample collection, handling, DNA purification and sequencing are already available and evaluated $(4,13)$, but specific choices have to be agreed so that the process is fully standardized - ensuring balanced representation from all bacterial species, maximising read quality and test sensitivity are key issues. Bioinformatics methods for generating AMR gene abundance data are already available (4) but, again, specific choices need to be made. Unlike the sequencing step, here the choice is not irrevocable; metagenomics data, once generated, can be re-analysed when new bioinformatics methods become available or reference sequence databases are updated (allowing, for example, retrospective study of the spread of newly identified resistance genes).

In addition, agreements are needed on sample and data sharing that comply with the Nagoya Protocol to the Convention on Biological Diversity (14). Agreement is also needed on a standardised 
data reporting format, noting that gene abundance data are different in nature from isolate-based data. Competent national and global authorities must be identified. The sewage-based program must be integrated with existing isolate-based surveillance programs. A formal economic analysis is necessary and a case for affordability and sustainability needs to be made.

It is important to ensure that global sewage-based surveillance is adopted by the right international organization(s) with the mandate to perform regional and/or global AMR surveillance, such as WHO and, for Europe, ECDC. This would also ensure direct and sustainable links to existing surveillance systems such as GLASS. This does not, however, restrict national institutions from setting up national sewage-based surveillance at any stage.

An immediate working model for global surveillance could be annual collection of sewage samples across the globe, with shipment of sewage to a central facility, perhaps a WHO Collaborating Centre, responsible for the subsequent sequencing, bioinformatics, analyses, and reporting (Fig. 1). As capacity builds around the world this could transition into a system where DNA is purified and sequenced and perhaps also analysed locally and subsequently shared globally via an international agency.

Standard reporting should as a minimum include AMR gene abundances per country over time for each antimicrobial class. Reporting frameworks will need to be adapted to accommodate this different kind of data. Though we recognise that there may be political sensitivities, we would strongly encourage global public sharing of the raw data with the global research community wherever possible, taking advantage of the global repositories for sharing sequencing data already in place.

In our opinion, the implementation of a global sewage-based AMR surveillance system would have substantial and rapid benefits, especially in resource-poor settings. It could be quickly implemented at a comparatively very low cost. By providing population-level information it would complement and augment current AMR surveillance efforts, so contributing to meeting the key objectives of AMR surveillance at a global scale.

References and Notes:

1. World Health Organization. 2014. Antimicrobial resistance: global report on surveillance. (http://apps.who.int/iris/bitstream/10665/112642/1/9789241564748 eng.pdf).

2. World Bank Group. 2017. Resistant infections; A Threat to Our Economic Future. (http://documents.worldbank.org/curated/en/323311493396993758/pdf/final-report.pdf).

3. World Bank Group. 2019. Pulling Together to Beat Superbugs; Knowledge and Implementation Gaps in Addressing Antimicrobial Resistance.

(http://documents.worldbank.org/curated/en/430051570735014540/pdf/Pulling-Together-to-BeatSuperbugs-Knowledge-and-Implementation-Gaps-in-Addressing-Antimicrobial-Resistance.pdf).

4. $\quad$ R. S. Hendriksen et al., Nat Commun. 10, 1124 (2019).

5. J. Q. Su et al., Microbiome 5, 84 (2017).

6. United Nations, Department of Economic and Social Affairs, Population Division (2019). World Urbanization Prospects: The 2018 Revision (ST/ESA/SER.A/420). New York: United Nations. https://population.un.org/wup/Publications/Files/WUP2018-Report.pdf (last accessed January 6th, 2020).

7. K. Acharya et al., Sci Rep 9, 15726 (2019).

8. M. R. Perry, D. McClean, C. Simonet, M. Woolhouse, L McNally, Nature Ecology and Evolution (in press).

9. $\quad$ S. M. Joseph et al., mSystems 4, e00327-19 (2019). 
10. K. M. M. Pärnänen et al., Sci Adv. 5, eaau9124 (2019).

11. M. Hutinel et al., Euro Surveill 24, (2019).

12. R. S. Hendriksen et al., PLoS One 14, e0222531 (2019).

13. A. D. Li et al., FEMS Microbiol Ecol 94, (2018).

14. C. Dos S Ribeiro, M. P. Koopmans, G. B. Haringhuizen, Science 362, 404-406 (2018).

\section{Acknowledgements:}

We are grateful to the WHO GLASS team for helpful discussions and to three anonymous reviewers for constructive comments on an earlier draft. The Global Sewage Surveillance Project is supported by The Novo Nordisk Foundation (NNF16OC0021856: Global Surveillance of Antimicrobial Resistance). The authors contributed equally to this work. FA declares that he is the current head of a WHO Collaborating Centre for AMR.

Figure 1. Comparison of current clinical surveillance and sewage-based surveillance. Clinical, isolate-based surveillance is based on small numbers of samples mainly from patients in health-care facilities that are tested locally for resistance to selected antibiotics using standard microbiological methods. Sewage-based surveillance is based on pooled samples from very large (typically 4500,000 ), mainly healthy populations that are tested locally or centrally for all known resistance genes using sequencing and bioinformatics analysis. 